Cahiers Charlevoix

Études franco-ontariennes
Cahiers Charlevoix Études franco-ontariennes

or Crevenerix of

\title{
Transferts linguistiques et anglicisation des francophones. Les enjeux de l'exogamie au Canada
}

\section{Roger Bernard}

Volume 2, 1997

URI : https://id.erudit.org/iderudit/1039456ar

DOI : https://doi.org/10.7202/1039456ar

Aller au sommaire du numéro

\section{Éditeur(s)}

Société Charlevoix

Presses de l’Université d'Ottawa

ISSN

1203-4371 (imprimé)

2371-6878 (numérique)

Découvrir la revue

Citer cet article

Bernard, R. (1997). Transferts linguistiques et anglicisation des francophones. Les enjeux de l'exogamie au Canada. Cahiers Charlevoix, 2, 213-262.

https://doi.org/10.7202/1039456ar
Résumé de l'article

Roger Bernard approfondit ses recherches sur " les enjeux de l'exogamie » et la vulnérabilité des groupes minoritaires. À partir d'une compilation plus détaillée des données du recensement de 1991, il analyse les conséquences de ce phénomène sur les transferts linguistiques en englobant, dans son propos, les foyers mixtes francophones et les foyers mixtes anglophones. Cette nouvelle étude, qui prend en compte les groupes d'âges des épouses de langue maternelle française, lui permet de réviser et de nuancer les résultats obtenus dans son article précédent, et de faire les liens qui s’imposent entre exogamie et anglicisation : " plus le milieu est minoritaire, observe-t-il, plus les taux d'exogamie sont élevés, et plus les taux d'anglicisation des parents et des enfants sont élevés "; ce qui l'amène à l'évidence que l'exogamie est une cause majeure de l'anglicisation des minorités françaises au Canada. 


\section{TRANSFERTS LINGUISTIQUES ET ANGLICISATION DES FRANCOPHONES. LES ENJEUX DE L'EXOGAMIE AU CANADA}

Roger Bernard

Faculté d'éducation

Université d'Ottawa, Ottawa

Cahiers Charlevoix 2, 1997, pp. 213-262. 
SOMMAIRE

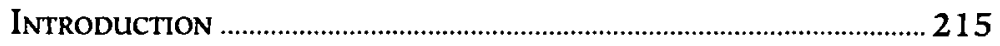

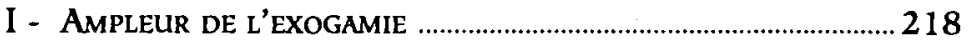

II - Passage de l'endogamie À l'exogamie ………..........................232

1. Endogamie: une norme sociale contraignante

2. Exogamie: une nouvelle structure familiale

III - EXogamie ET ANGLICISATION

1. Anglicisation des partenaires francophones

2. Anglicisation des enfants

3. Une relation explicative complexe

Conclusion. 


\section{TRANSFERTS LINGUISTIQUES ET ANGLICISATION DES FRANCOPHONES. LES ENJEUX DE L'EXOGAMIE AU CANADA ${ }^{1}$}

\section{INTRODUCTION}

À l'époque de l'ouverture à l'autre, de la mondialisation des perspectives et de l'interpénétration culturelle qui doivent conduire au métissage identitaire, l'exogamie, le mariage à l'extérieur de son groupe d'appartenance, pose encore des problèmes, tant au niveau individuel que collectif ${ }^{2}$. De façon générale, plus la vitalité ethnoculturelle d'un groupe est faible, plus les enjeux de l'exogamie sont grands; paradoxalement, plus la vitalité d'un groupe est faible, plus grandes sont les probabilités que les taux d'exogamie soient élevés. Si au Canada, comme le démontrent plusieurs études réalisées au

' Je voudrais remercier Christine Lebel, étudiante au doctorat en éducation à l'Université d'Ottawa, pour son aide précieuse dans la préparation de la version finale de ce texte.

${ }^{2}$ E. L. Cerroni-Long, "Marrying Out: Socio-Cultural and Psychological Implications of Intermarriage», Journal of Comparative Family Studies, XVI(1), 1985, pp. 25-44. 
cours des dernières années, notamment celles de Charles Castonguay ${ }^{3}$ et de Réjean Lachapelle ${ }^{4}$, l'exogamie conduit aux transferts linguistiques et à l'anglicisation, les groupes les plus minoritaires sont les plus vulnérables et les plus menacés. C'est à partir des données du recensement de 1991 de Statistique Canada que nous tenterons surtout de faire la lumière sur les conséquences de l'exogamie en regard des transferts linguistiques, en d'autres mots, d'analyser les enjeux de l'exogamie en ce qui a trait au processus d'anglicisation des minorités canadiennesfrançaises et acadiennes.

La société canadienne, en valorisant le bilinguisme, le pluralisme et le multiculturalisme, favorise l'exogamie. En se présentant comme une société ouverte à l'autre, elle veut faire tomber, dans la mesure du possible, les barrières entre les groupes cultu-

\footnotetext{
${ }^{3}$ Charles Castonguay, "Les Transferts linguistiques au foyer", Recherches sociographiques, vol. XVII, nं 3, 1976, pp. 341-351; id., «Exogamie et anglicisation chez les minorités canadiennes-françaises», La Revue canadienne de sociologie et d'anthropologie, vol. 16, n' 1, 1979, pp. 21 31; id., "L'Exogamie précoce et la prévision des taux de transfert linguistique», Recherches sociographiques, vol. XX, n 3,1979 , pp. 403408; id., "L'Orientation linguistique des marlages mixtes dans la région de Montréal", Recherches sociographiques, vol. XXI, n` 3, 1980, pp. 225-251; id., «Intermarriage and Language Shift in Canada, 1971 and 1976", Canadian Journal of Sociology, vol. 7, n 3, 1982, pp. 263277; id., L'Évolution de l'exogamie et de ses incidences sur les transferts linguistiques chez les populations provinciales de langue maternelle française au Canada entre 1971 et 1981, Québec, Le Conseil de la langue française, 1983.

${ }^{4}$ Réjean Lachapelle et Jacques Henripin, La Situation démolinguistique au Canada, Montréal, L'Institut de Recherches politiques, 1980; Réjean Lachapelle, Evolution des groupes linguistiques de langues officielles $d u$ Canada. Tendances démolinguistiques et évolution des institutions canadiennes, Ottawa, Secrétariat d'État du Canada, 1989, pp. 7-34; id., L'Exogamie dans les populations francophones en situation minoritaire, communication au colloque national sur l'exogamie, Rockland (Ontario), 1994.
} 
rels et ainsi affaiblir les frontières ethniques susceptibles de créer des enclaves qui débouchent sur l'exclusion au détriment de l'inclusion. Nonobstant ces idéaux de cohabitation individuelle et d'accommodation communautaire, l'endogamie représente encore la norme d'organisation familiale pour la très grande majorité des foyers canadiens. Le taux d'exogamie varie considérablement d'un groupe culturel à l'autre, les variations étant surtout fonction de la taille du groupe et de son poids démographique; habituellement, plus le groupe est grand et son poids démographique imposant, plus le taux d'exogamie sera faible. Si le taux d'exogamie des époux et des épouses de langue française est faible au Canada et que l'endogamie est encore la norme, il faut voir que cette moyenne nationale masque une tout autre réalité lorsque nous considérons le poids démographique régional ou provincial de ces derniers. En effet, si le taux d'exogamie des francophones est très faible au Québec et au Nouveau-Brunswick, en revanche il est élevé en Ontario et très élevé en Colombie-Britannique.

Il faut toutefois demeurer prudent quant aux explications simples ou trop rapides. L'exogamie est un phénomène social complexe qui doit être replacé dans son contexte historique pour mieux le cerner. De plus, la question des mariages mixtes au Canada est encore un sujet épineux puisque cette problématique est tour à tour individuelle et collective, religieuse et linguistique, culturelle et sociale. Lorsque nous tentons de lier l'anglicisation et la faible vitalité ethnoculturelle des minorités canadiennes-françaises aux mariages entre anglophones et francophones, la question de l'exogamie devient une affaire délicate qui illustre très bien l'effet pervers d'agrégation ${ }^{5} d^{\prime} u n e$

${ }^{5}$ Raymond Boudon, La Logique du social, Paris, Hachette, 1979, 275 p. 
décision amoureuse singulière qui se voulait dans bien des cas la réalisation d'un rêve, soit celui de créer une institution bilingue pour encadrer le bilinguisme individuel au sein d'un pays qui reconnaît et défend la légitimité de la dualité linguistique. Cette décision particulière, raisonnable selon la logique amoureuse et souhaitable selon l'idéologie dominante du bilinguisme, entraîne des effets collectifs non voulus et amplifiés au plan social. Cet effet d'agrégation n'était pas recherché par les parents en situation d'exogamie qui souvent espéraient le contraire: que le mariage mixte conduise à la vitalité et au renouvellement de la communauté canadienne-française.

L'exogamie est donc un sujet démographique fascinant et complexe qui exige une analyse globale prenant en compte le sentiment amoureux, la liberté du sujet, l'autonomie des personnes ainsi que l'étude de phénomènes sociétaux macrosociologiques qui favorisent l'éclosion de l'exogamie comme nouvelle forme d'organisation familiale. De l'agrégation d'actions individuelles, il résulte toutefois un effet collectif pervers et contraire à celui sous-jacent à la prise de décision initiale des conjoints exogames soucieux d'assurer le maintien de la communauté minoritaire.

\section{I - Ampleur de l'exogamie}

Les données du tableau 1 montrent l'ampleur de l'exogamie des époux et des épouses de langue maternelle française dans les différentes provinces canadiennes en 1971, en 1986 et en $1991^{6}$. En tenant

${ }^{6}$ Il faut toutefois retenir que la comparaison des données linguistiques des derniers recensements appelle la prudence d'interprétation parce que les questions ou les directives ont été modifiées et que la méthode de répartition des réponses multiples a fait l'objet de transformations et d'ajustements. 
compte des difficultés de comparaison des statistiques d'un recensement à l'autre, malgré tout il ressort assez clairement des données du premier tableau que les taux d'exogamie sont sensiblement les mêmes, qu'il s'agisse des époux ou des épouses de langue maternelle française. En effet, au Canada sans le Québec, 39,3\% des épouses francophones se retrouvent dans une famille exogame, comparativement à $37 ; 3 \%$ des époux en 1991. Lorsque nous considérons le Canada sans l'Ontario, le Québec et le Nouveau-Brunswick, le taux d'exogamie se situe à $56,4 \%$ pour les épouses francophones, alors qu'il est de $57,2 \%$ pour les époux.

La deuxième grande constatation concerne la hausse significative des taux d'exogamie entre 1971 et 1991. À Terre-Neuve, les taux d'exogamie ont grimpé de plus de vingt points (\%) durant cette période, alors qu'en Ontario l'augmentation est de dix points (\%), une augmentation moins spectaculaire, mais tout de même importante. Au NouveauBrunswick, l'augmentation est plus modeste, se situant entre trois et quatre points. Il y a donc, d'une décennie à l'autre, augmentation du taux d'exogamie chez les époux et les épouses francophones du Canada.

La troisième grande constatation découle directement de la deuxième: à travers le temps, les augmentations sont plus prononcées dans les provinces ou les régions où le poids démographique des francophones est le plus faible. Nous pourrions ainsi affirmer que plus les francophones sont minoritaires, plus ils sont enclins à l'exogamie, de telle sorte que plus le processus de minorisation s'accentue d'un recensement à l'autre, plus le taux d'exogamie a tendance à grimper rapidement. En 1991, plus du tiers des époux et des épouses de langue maternelle française 
qui vivent à l'extérieur du Québec se retrouvent dans une famille exogame et cette proportion monte à six sur dix $(6 / 10)$ lorsque nous considérons le Canada sans le Québec, l'Ontario et le Nouveau-Brunswick. Cette même année, le taux d'exogamie des époux et des épouses de langue française de la ColombieBritannique était de $71,2 \%$, alors qu'au NouveauBrunswick il oscillait autour de $13,7 \%$. Nous retrouvons alors une proportion plus élevée de mariages hétérolinguistiques que de mariages homolinguistiques chez les conjoints francophones dans les provinces et les régions suivantes: Colombie-Britannique, Alberta, Saskatchewan, Terre-Neuve, Yukon et les Territoires du Nord-Ouest. Au Manitoba, en Ontario, à l'île-duPrince-Édouard et en Nouvelle-Écosse, les taux d'exogamie des époux et des épouses francophones varient de $40,9 \%$ à $45,6 \%$.

La quatrième constatation permet donc d'affirmer que plus le poids démographique des francophones est faible, plus son taux d'exogamie est élevé. En raison des processus de minorisation et de dispersion, les tendances démographiques deviennent de plus en plus lourdes et déterminantes, confirmant alors l'hypothèse liant le poids démographique et l'exogamie. À la lumière de ces constatations, il ressort que le taux d'exogamie chez les francophones est inversement proportionnel à leur poids démographique.

En guise de récapitulatif nous pourrions affirmer qu'en situation très minoritaire, le taux d'exogamie est très souvent supérieur au taux d'endogamie alors qu'en situation majoritaire, l'exogamie est un phénomène marginal en regard de la prédominance de l'endogamie. 


\section{TABLEAU 1}

Taux d'exogamie des époux et des épouses de langue maternelle française, provinces et Canada, 1971, 1986' et $1991^{8}$.

\begin{tabular}{|c|c|c|c|c|c|c|c|}
\hline \multirow[b]{2}{*}{ Provinces } & \multicolumn{3}{|c|}{$\begin{array}{l}\text { Epoux de } \\
\text { langue } \\
\text { maternelle } \\
\text { franģaise }\end{array}$} & \multicolumn{3}{|c|}{$\begin{array}{l}\text { Epouse de } \\
\text { langue } \\
\text { maternelle } \\
\text { française }\end{array}$} & \multirow{2}{*}{$\begin{array}{c}\text { Epoux et } \\
\text { épouse de } \\
\text { langue mat } \\
\text { française } \\
1991\end{array}$} \\
\hline & 1971 & 1986 & 1991 & 1971 & 1986 & 1991 & \\
\hline \multicolumn{8}{|l|}{ Colombie- } \\
\hline Britannique & 61,1 & 67,6 & 73,1 & $\begin{array}{l}58,5 \\
476\end{array}$ & $\begin{array}{l}68,1 \\
579\end{array}$ & 70,8 & $\begin{array}{l}71,2 \\
615\end{array}$ \\
\hline $\begin{array}{l}\text { Alberta } \\
\text { Saskatchewan }\end{array}$ & $\begin{array}{l}47,3 \\
42,3\end{array}$ & $\begin{array}{l}55,9 \\
53,0\end{array}$ & $\begin{array}{l}62,1 \\
58,1\end{array}$ & $\begin{array}{l}47,6 \\
42,0\end{array}$ & $\begin{array}{l}57,9 \\
54,7\end{array}$ & $\begin{array}{l}61,0 \\
57,6\end{array}$ & $\begin{array}{l}61,5 \\
57,8\end{array}$ \\
\hline Manitoba & 32,4 & 41,0 & 45,3 & 32,8 & 40,4 & 46,0 & 45,6 \\
\hline Ontario & 28,9 & 37,8 & 38,8 & 30,4 & 34,4 & 42,9 & 40,9 \\
\hline $\begin{array}{l}\text { Québec } \\
\text { Nouveau- }\end{array}$ & - & 3,4 & 3,7 & - & 2,7 & 4,8 & - \\
\hline $\begin{array}{l}\text { Brunswick } \\
\text { Île-du-Prince- }\end{array}$ & 9,1 & 11,3 & 13,3 & 10,9 & 10,8 & 14,1 & 13,7 \\
\hline Édouard & 23,6 & 39,5 & 42,6 & 28,7 & 37,3 & 44,8 & 43,5 \\
\hline Nouvelle-Écosse & 32,6 & 39,6 & 46,1 & 32,1 & 40,5 & 44,4 & 45,3 \\
\hline $\begin{array}{l}\text { Terre-Neuve } \\
\text { Territoires du }\end{array}$ & 45,5 & 57,9 & 65,8 & 38,0 & 61,5 & 62,2 & 63,9 \\
\hline Nord-Ouest & - & 58,2 & $68,6^{9}$ & - & 65,7 & $61,6^{10}$ & 63,8 \\
\hline Yukon & - & 60,7 & 68,6 & - & 69,4 & 61,6 & 67,0 \\
\hline $\begin{array}{l}\text { Canada (sans Ont } \\
\text { Qc, N.-B.) } \\
\text { Canada (sans le }\end{array}$ & t., & & 57,2 & - & - & 56,4 & - \\
\hline Québec) & - & 34,8 & 37,3 & - & 33,1 & 39,3 & 38,3 \\
\hline Canada & - & 8,2 & 8,8 & - & 7,3 & 10,2 & - \\
\hline
\end{tabular}

SOURCES"I

${ }^{7}$ En 1986, seuls les mariages avec un partenaire ayant déclaré l'anglais ou une autre langue comme seule langue maternelle ont été considérés. ${ }^{8}$ En 1991, seuls les époux et les épouses de langue maternelle française unique ont été considérés, mais les partenaires exogames pouvalent être de langue maternelle anglaise ou d'une autre langue maternelle. ${ }^{9}$ Les données relatives aux Territoires du Nord-Ouest incluent celles du Yukon pour l'année 1991.

${ }^{10}$ Les données relatives au Yukon incluent celles des Territoires du Nord-Ouest pour l'année 1991.

${ }^{11}$ Roger Bernard, Le Choc des nombres. Dossier statistique sur la francophonie canadienne, 1951-1986. Ottawa, Fédération des jeunes 
Le deuxième tableau met en relation le taux d'exogamie et le groupe d'âge de l'épouse de langue maternelle française (unique) selon la province de résidence. Lorsque nous considérons le Canada sans le Québec, l'Ontario et le Nouveau-Brunswick, en l'occurrence les provinces où le poids démographique des francophones est très faible, nous constatons que plus de la moitié des épouses francophones de moins de 55 ans vivent dans une situation de mariage mixte et que cette proportion grimpe à près des deux tiers pour les épouses francophones de moins de 25 ans. En Colombie-Britannique, où les taux d'exogamie sont les plus élevés, trois épouses francophones sur quatre de moins de $\mathbf{4 5}$ ans se trouvent en situation d'exogamie, alors qu'en Alberta cette proportion se situe à deux sur trois pour le même groupe d'âge. En Colombie-Britannique, tous les groupes d'âge ont un taux d'exogamie supérieur à $60 \%$, tandis qu'en Alberta cette proportion s'applique aux femmes de moins de 55 ans et qu'en Saskatchewan, le taux fluctue de $58,8 \%$ à $70,1 \%$ pour les femmes francophones des mêmes groupes d'âge. Au Manitoba, les taux d'exogamie sont plus élevés que les taux d'endogamie pour les femmes francophones de moins de 45 ans, mais ces taux baissent considérablement pour les femmes plus âgées, passant de $44 \%$ pour les 45 à 54 ans à $27,7 \%$ pour celles de plus de 65 ans. À l'île-du-Prince-Édouard et en Nouvelle-Écosse, la tendance est très près de celle que nous retrouvons au Manitoba, alors qu'à Terre-

Canadiens français, 1990, 311 p.; Louise Dallaire et Réjean Lachapelle, Profils linguistiques des communautés de langues officielles, Ottawa, Secrétariat d'État du Canada, 1990 (13 volumes); Fédération des francophones hors Québec, Les Héritiers de Lord Durham, vol. 1, 1977, 125 p.; Réjean Lachapelle, L'Exogamie dans [...], op. cit. 
Neuve les taux d'exogamie observés se rapprochent de ceux notés en Saskatchewan. En situation très minoritaire, les taux d'exogamie varient de façon marquée d'une tranche d'âge à l'autre: modérés chez les épouses âgées, mais très élevés chez les plus jeunes.

\section{TABLEAU 2}

Taux d'exogamie des épouses de langue maternelle française (unique) vivant dans une famille époux-épouse selon le groupe d'âge de l'épouse, provinces et Canada, 1991.

\begin{tabular}{|c|c|c|c|c|c|c|}
\hline \multirow[b]{2}{*}{ Provinces } & \multicolumn{6}{|c|}{ Groupes d'age } \\
\hline & $15-24$ & 25-34 & $35-44$ & 45-54 & $55-64$ & $65+$ \\
\hline Colomble-Britannique & 74,0 & 73,6 & 75,6 & 69,8 & 65,6 & 62,4 \\
\hline Alberta & 67,1 & 66,1 & 65,7 & 60,6 & 50,9 & 42,4 \\
\hline Saskatchewan & 65,7 & 70,1 & 68,6 & 58,8 & 47,1 & 37,3 \\
\hline Manitoba & 57,4 & 54,7 & 52,5 & 44,0 & 36,6 & 27,7 \\
\hline Ontario & 46,5 & 47,1 & 45,9 & 41,8 & 35,9 & 33,0 \\
\hline Québec & 4,8 & 4,9 & 5,0 & 5,0 & 4,7 & 4,3 \\
\hline Nouveau-Brunswick & 15,9 & 15,1 & 15,1 & 14,1 & 12,2 & 9,5 \\
\hline Île-du-Prince-Edouard & 61,5 & 55,1 & 53,8 & 45,3 & 31,3 & 25,0 \\
\hline Nouvelle-Écosse & 51,1 & 51,1 & 50,3 & 44,0 & 39,2 & 26,2 \\
\hline Terre-Neuve & 57,1 & 63,9 & 71,4 & 56,0 & 57,1 & 41,7 \\
\hline T.N.-O. et Yukon & 100,0 & 61,0 & 60,0 & 56,3 & 71,4 & 50,0 \\
\hline Canada (sans $Q \mathcal{c}$, Ont., N.-B.) & 63,4 & 62,5 & 62,8 & 55,8 & 48,5 & 39,5 \\
\hline Canada (sans le Québec) & 41,5 & 42,4 & 42,3 & 39,4 & 34,3 & 29,3 \\
\hline Canada & 9,5 & 10,4 & 10,9 & 10,6 & 9,4 & 8,6 \\
\hline
\end{tabular}

SOURCE $^{12}$

Dans les trois autres provinces, le Québec, l'Ontario et le Nouveau-Brunswick, les taux d'exogamie fluctuent d'une province à l'autre en fonction du poids démographique de la population francophone. En Ontario, pour les épouses francophones de moins de 55 ans, les taux d'exogamie sont légèrement

${ }^{12}$ Recensement du Canada de 1991. 
inférieurs aux taux d'endogamie, variant de $41,8 \%$ à $47,1 \%$, alors qu'ils oscillent autour de $33 \%$ pour les femmes de 55 ans et plus. Au Nouveau-Brunswick, ces taux sont relativement plus faibles que dans les autres provinces canadiennes-anglaises, s'y situant à $14,1 \%$ pour les femmes de 45 à 54 ans et à $15,9 \%$ pour les femmes de moins de 25 ans. Nous pouvons aussi remarquer qu'en Ontario et au NouveauBrunswick, les écarts entre les taux d'un groupe d'âge à l'autre sont moins prononcés que dans les autres provinces où les francophones sont plus minoritaires. Toutefois, la tendance générale demeure la même: plus les femmes francophones sont jeunes, plus les taux d'exogamie sont élevés, et plus le poids démographique des francophones est faible, plus la relation entre les deux variables semble forte. Au Québec, la situation est très différente: les taux d'exogamie sont très faibles, se situant à une femme francophone sur vingt $(1 / 20)$ et sont très stables d'un groupe d'âge à l'autre, soit de $4,8 \%$ pour les femmes de moins de 25 ans et $4,7 \%$ pour les femmes ayant entre 55 et 64 ans.

Ainsi, au regard de l'ensemble de ces données au Canada, il devient évident que l'endogamie représente la norme pour la très grande majorité des femmes de langue maternelle française, soit 9 sur 10 , mais cette proportion canadienne cache une réalité très différente lorsque nous considérons les données provinciales qui révèlent que, dans une majorité de provinces, c'est l'exogamie qui prédomine. Outre ces constatations, il ressort que plus les francophones sont minoritaires, plus les écarts entre les taux d'exogamie d'un groupe d'âge sont élevés par opposition aux situations majoritaires où il y a stabilité de ces taux, d'un groupe d'âge à l'autre. 
Dans cette perspective, si nous reconnaissons qu'il y a amplitude des processus de minorisation et de dispersion des francophones dans plusieurs provinces canadiennes, nous pouvons conclure que les écarts des taux d'exogamie auront tendance à s'accentuer eu égard aux différences marquées entre les taux d'exogamie chez les jeunes épouses francophones par rapport aux taux relevés pour les épouses plus âgées. De plus, les tendances décelées au tableau 2 recoupent celles du premier tableau: d'un recensement à l'autre, donc de 1971 à 1991, les taux d'exogamie augmentent fortement dans les provinces où les francophones sont minoritaires et, plus ces derniers sont minoritaires, plus ces augmentations sont considérables. Également, plus les épouses francophones sont jeunes, plus les taux d'exogamie sont élevés ce qui permet d'envisager le fait que ces tendances démographiques sont décisives et marquantes.

En comparant les données du tableau 3 présentant les taux d'exogamie des époux de langue maternelle française selon les différents groupes d'âge, à celles du tableau 2, nous remarquons qu'il n'y a pas de différences majeures qui ressortent de telle sorte que les taux d'exogamie varient considérablement d'une tranche d'âge à l'autre. Ainsi ils sont modérés autant chez les épouses que chez les époux âgés, mais très élevés chez les plus jeunes, qu'il s'agisse des époux ou des épouses.

Toutefois, nous pouvons remarquer lorsque nous considérons le Canada dans son ensemble, que les taux d'exogamie des époux sont légèrement plus bas que ceux des épouses et ce, pour tous les groupes d'âge. Cette tendance s'applique aussi à l'Ontario au Québec et à l'île-du-Prince-Édouard. Lorsque nous 
considérons le Canada sans le Québec, cette même tendance apparaît: les taux d'exogamie des époux sont plus bas que ceux des épouses pour tous les groupes d'âge à l'exception de ceux de la cohorte de 15 à 24 ans. Par ailleurs, pour le Canada francophone très minoritaire (sans l'Ontario, le Nouveau-Brunswick et le Québec), nous observons une différence: les taux d'exogamie sont plus élevés chez les époux comparativement aux épouses pour les groupes d'âge inférieurs à 45 ans, alors qu'ils sont inférieurs pour ceux de 45 et plus. Cette tendance s'accentue dans l'ensemble des provinces où les francophones sont très minoritaires, notamment en Colombie-Britannique, en Alberta, en Saskatchewan et au Manitoba.

\section{TABLEAU 3}

Taux d'exogamie des époux de langue maternelle française (unique) vivant dans une famille époux-épouse selon le groupe d'âge de l'épouse, provinces et Canada, 1991.

Provinces

Colombie-Britannique

Alberta

Saskatchewan

Manitoba

Ontario

Québec

Île-du-Prince-Édouard

Nouvelle-Écosse

Terre-Neuve

T.N.-O. et Yukon

Canada (sans Qc, Ont., N.-B.)

Canada (sans Qc)

Canada
Nouveau-Brunswick

\section{Groupes d'âge}

15-24 25-34 35-44 45-54 55-64 65+

$\begin{array}{rrrrrr}\mathbf{7 9 , 3} & \mathbf{7 7 , 0} & \mathbf{7 5 , 6} & \mathbf{6 9 , 6} & \mathbf{6 3 , 1} & \mathbf{6 0 , 1} \\ \mathbf{7 2 , 9} & \mathbf{7 0 , 0} & \mathbf{6 6 , 9} & \mathbf{5 7 , 1} & \mathbf{4 7 , 6} & \mathbf{4 1 , 3} \\ \mathbf{6 7 , 6} & \mathbf{7 1 , 7} & \mathbf{7 0 , 4} & \mathbf{5 8 , 8} & \mathbf{4 3 , 9} & \mathbf{3 5 , 7} \\ \mathbf{6 1 , 9} & \mathbf{5 5 , 3} & \mathbf{5 3 , 5} & \mathbf{4 1 , 4} & \mathbf{3 0 , 8} & \mathbf{2 6 , 2} \\ \mathbf{4 3 , 4} & \mathbf{4 4 , 6} & \mathbf{4 2 , 6} & \mathbf{3 5 , 9} & \mathbf{3 0 , 8} & \mathbf{2 7 , 0} \\ \mathbf{4 , 2} & \mathbf{4 , 2} & \mathbf{3 , 8} & \mathbf{3 , 4} & \mathbf{2 , 9} & \mathbf{3 , 1} \\ 1 \mathbf{7 , 8} & 15,6 & 14,1 & 12,4 & \mathbf{9 , 9} & \mathbf{7 , 4} \\ \mathbf{5 8 , 3} & \mathbf{5 4 , 4} & \mathbf{5 0 , 7} & \mathbf{4 5 , 3} & \mathbf{2 9 , 8} & \mathbf{1 8 , 8} \\ \mathbf{5 6 , 1} & \mathbf{5 4 , 8} & \mathbf{5 2 , 4} & \mathbf{4 4 , 8} & \mathbf{3 7 , 7} & \mathbf{2 4 , 1} \\ \mathbf{5 7 , 1} & \mathbf{6 9 , 0} & \mathbf{7 6 , 7} & \mathbf{5 9 , 3} & \mathbf{5 7 , 1} & \mathbf{4 6 , 2} \\ \mathbf{1 0 0} & \mathbf{6 3 , 6} & \mathbf{7 1 , 4} & \mathbf{6 6 , 7} & \mathbf{6 6 , 7} & \mathbf{6 6 , 7} \\ \mathbf{6 8 , 8} & \mathbf{6 5 , 6} & \mathbf{6 3 , 8} & \mathbf{5 4 , 7} & 45,2 & 37,4 \\ \mathbf{4 2 , 2} & \mathbf{4 2 , 3} & \mathbf{4 0 , 7} & \mathbf{3 5 , 5} & \mathbf{3 0 , 0} & \mathbf{2 5 , 3} \\ \mathbf{9 , 2} & \mathbf{9 , 8} & \mathbf{9 , 6} & \mathbf{8 , 4} & \mathbf{7 , 1} & \mathbf{6 , 7}\end{array}$

SOURCE $^{13}$

${ }^{13}$ Recensement du Canada de 1991. 
Cependant, dans toutes les provinces à l'exception de Terre-Neuve, la différence entre les taux d'exogamie des époux âgés de plus de 65 ans par rapport à ceux de la première cohorte de 15 à 24 ans, est plus élevée chez les époux que chez les épouses. Il y a done plus de variabilité des taux selon les groupes d'âge, chez les époux que chez les épouses, ce qui nous conduit à affirmer que plus les francophones sont minoritaires, plus ces écarts sont grands.

En consultant les figures 1 et $2^{14}$, nous sommes amenés à réaliser qu'à travers le temps, les taux d'exogamie se déplaceront de la gauche vers la droite et que chaque génération en vieillissant gardera sensiblement le même taux d'exogamie puisque le taux suit la cohorte d'âge d'une génération à l'autre. Il est fort peu probable que les taux d'exogamie baissent à travers le temps. Il est donc facile d'envisager qu'à chaque recensement, si les tendances se maintiennent (et il n'y a pas lieu d'envisager le contraire), que les taux d'exogamie continueront d'augmenter sensiblement parce que, d'une cohorte à l'autre, les taux les plus bas, soit ceux du groupe d'âge de 65 ans et plus, seront remplacés par ceux de la nouvelle génération de gens nouvellement mariés qui auront fort assurément les taux les plus élevés de tous les groupes d'âge. Dans quelques décennies, dans l'ensemble des provinces canadiennes, à l'exception du Québec, de l'Ontario et du Nouveau-Brunswick, les taux d'exogamie, de tous les groupes d'âge des épouses et des époux de langue maternelle française, seront supérieurs aux taux d'endogamie et, de génération en génération, l'écart entre les deux taux risque de

\footnotetext{
${ }^{14}$ Les graphiques présentés aux figures 1 et 2 permettent de visualiser de façon instantanée les tendances contenues dans les tableaux 2 et 3 et présentées dans l'analyse qui précède.
} 
s'agrandir. En Ontario, les taux d'exogamie frôleront le $50 \%$, alors qu'au Nouveau-Brunswick, ils se rapprocheront du $20 \%$. Il n'y a qu'au Québec que les taux seront relativement stables et faibles, soit autour de $5 \%$ si les tendances démographiques se maintiennent (et il n'y a pas lieu ici non plus d'envisager le contraire).

\section{FIGURE $1^{15}$}

Taux d'exogamie des épouses de langue maternelle française (unique) vivant dans une famille époux-épouse selon le groupe d'âge de l'épouse, provinces du Canada, 1991.

\section{Pourcentages}

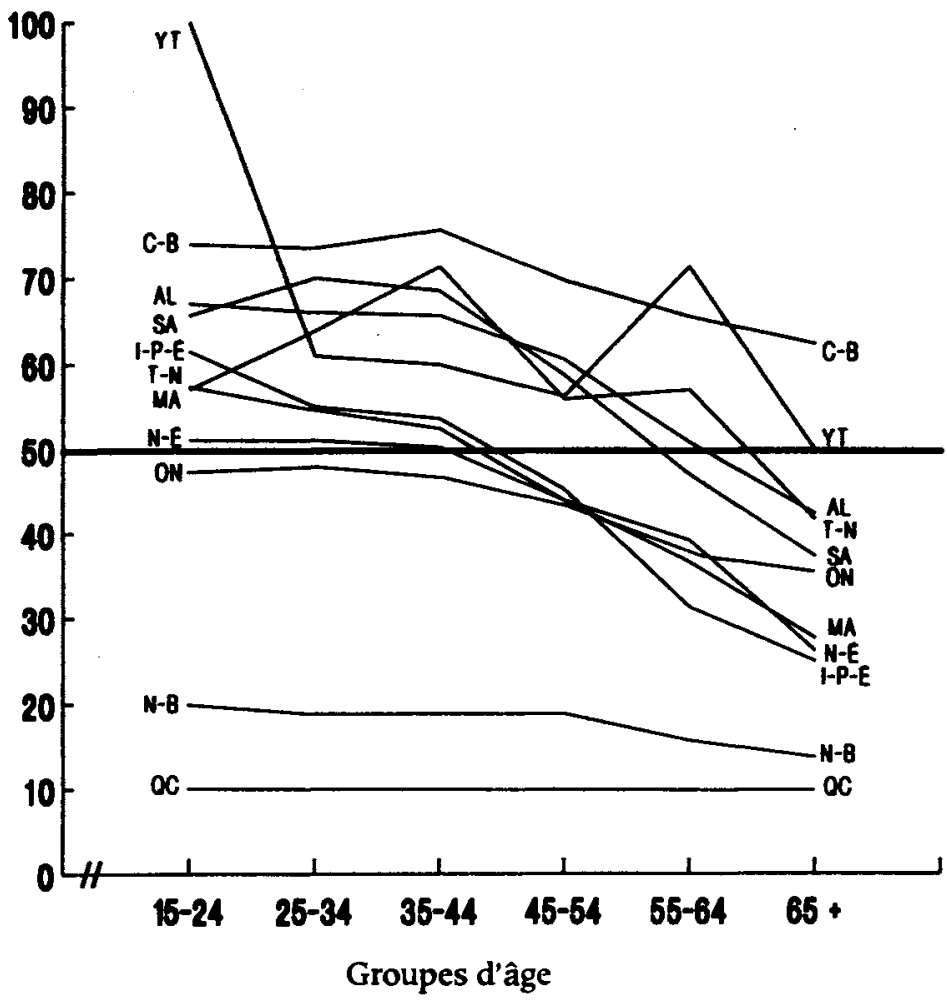

${ }^{15}$ Source: Recensement du Canada de 1991. 
FIGURE $2^{16}$

Taux d'exogamie des époux de langue maternelle française (unique) vivant dans une famille époux-épouse selon le groupe d'âge de l'épouse, provinces du Canada, 1991.

Pourcentages

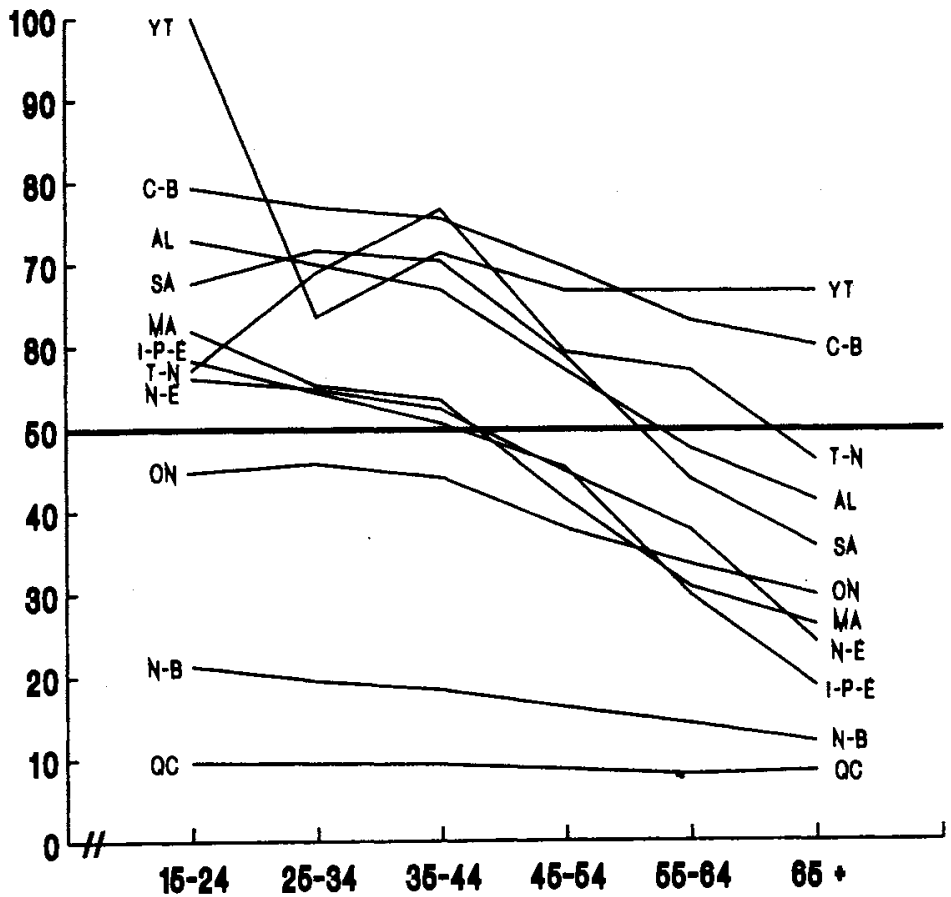

Groupes d'âge

Les tableaux 4 et 5 présentent les différentes situations d'endogamie et d'exogamie des époux et des épouses de langue maternelle française selon les différents groupes d'âge des épouses pour l'Ontario en 1991. Les données démontrent clairement que les taux d'endogamie sont plus faibles chez les cohortes

${ }^{16}$ Source: Recensement du Canada de 1991. 
plus jeunes que chez celles plus âgées et que ces taux sont plus élevés chez les époux que chez les épouses pour tous les groupes d'âge, la différence globale étant de $4 \%$. Pour les situations d'exogamie regroupant un conjoint francophone et anglophone, les taux sont aussi plus élevés pour les épouses que pour les époux. Il en va de même pour les situations d'exogamie regroupant un parent francophone et un parent allophone.

\section{TABLEAU 4}

Différentes situations d'endogamie et d'exogamie des épouses de langue maternelle française selon les groupes d'âge de l'épouse,

Ontario, 1991 (pourcentages).

\begin{tabular}{lccccc}
\hline $\begin{array}{l}\text { Groupes } \\
\text { d'âge }\end{array}$ & $\begin{array}{c}\text { Endogamie } \\
\text { Mère franç. } \\
\text { Père fr. }\end{array}$ & Total & $\begin{array}{c}\text { Mère fr. } \\
\text { Père an. }\end{array}$ & $\begin{array}{c}\text { Mère fr. } \\
\text { Père all. Père autre }\end{array}$ \\
$15-24$ & 53,5 & 46,5 & 41,1 & 3,8 & 1,7 \\
$25-34$ & 52,9 & 47,1 & 41,0 & 4,8 & 1,2 \\
$35-44$ & 54,1 & 45,9 & 38,2 & 6,4 & 1,4 \\
$45-54$ & 58,2 & 41,8 & 33,4 & 6,9 & 1,5 \\
$55-64$ & 64,1 & 35,9 & 28,7 & 5,5 & 1,7 \\
65 et plus & 67,0 & 33,0 & 27,2 & 4,0 & 1,9 \\
Total & 57,1 & 42,9 & 35,8 & 5,6 & 1,5 \\
\hline
\end{tabular}

SOURCE ${ }^{17}$

${ }^{17}$ Recensement du Canada de 1991. 
TABLEAU 5

Différentes situations d'endogamie et d'exogamie des époux de langue maternelle française selon les groupes d'âge de l'épouse, Ontario, 1991 (pourcentages).

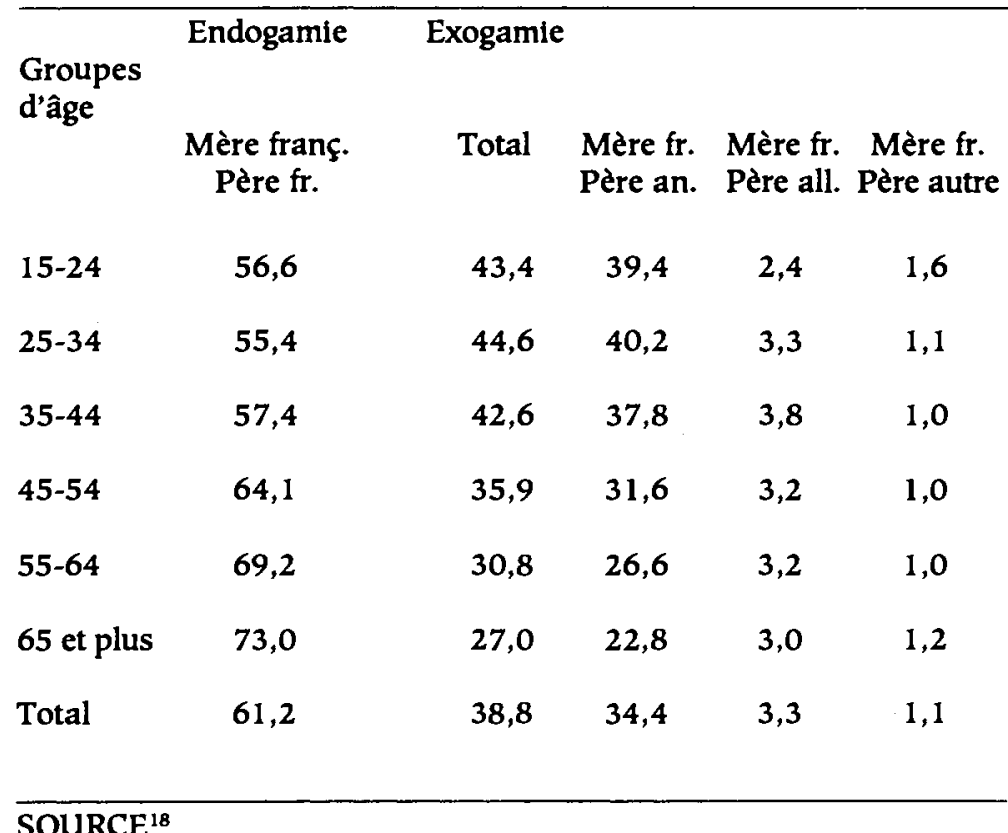

SOURCE $^{18}$

Dans les données qui précèdent (tableaux 1 à 5 et figures 1 et 2), nous avons brossé un portrait global des différentes situations d'endogamie et d'exogamie qui se développent à l'intérieur des communautés canadiennes-françaises depuis les dernières décennies. Nous avons donc été en mesure de constater que les taux d'exogamie augmentent d'une génération à l'autre, qu'ils sont considérablement plus élevés chez les jeunes mariés francophones et que ces taux sont légèrement plus faibles chez les époux que chez les épouses.

\footnotetext{
${ }^{18}$ Recensement du Canada de 1991.
} 
Ces phénomènes liés à la montée de l'exogamie depuis quelques générations et la prédiction que cette tendance se maintiendra chez les francophones canadiens en situation minoritaire, ne s'expliquent pas seulement par le profil démographique des communautés minoritaires. Car, tel que mentionné précédemment, l'exogamie est un phénomène complexe qui exige, pour bien le comprendre, d'être replacé dans son contexte historique et sociétal.

\section{II - Passage de l'endogamie a l'exogamie ${ }^{19}$}

\section{ENDOGAMIE: UNE NORME SOCIALE CONTRAIGNANTE}

En premier lieu, il importe de ne pas perdre de vue que c'est l'endogamie et non l'exogamie qui caractérise le type d'organisation familiale de l'ensemble de la société canadienne, et que l'endogamie est aussi la norme pour la très grande majorité des époux et des épouses francophones du Québec et de ceux qui se retrouvent dans des communautés francophones majoritaires. La montée et l'ampleur de l'exogamie, que nous avons décrites dans les pages qui précèdent, arrivent à un moment précis de l'histoire des francophones du Canada qui se retrouvent dans des conditions particulières qui favorisent cette nouvelle forme d'organisation familiale. La propension à l'exogamie n'est pas un phénomène propre aux francophones du Canada, car ces derniers se comportent comme la plupart des autres groupes culturels: en

\footnotetext{
${ }^{19}$ Une première version plus sommaire de cette analyse a été présentée dans Roger Bernard, "Langue maternelle et langue d'usage dans les foyers mixtes francophones. Les Enjeux de l'exogamie", Cahiers Charlevoix 1. Etudes franco-ontariennes, Sudbury, Prise de Parole et Société Charlevoix, 1995, pp. 241-289.
} 
situation majoritaire, c'est la tendance à l'endogamie qui prédomine nettement par opposition à une conjoncture minoritaire bien particulière qui les incite à l'exogamie. Ainsi, ils ne sont pas fondamentalement différents des autres communautés ethnoculturelles, certaines étant plus fermées, d'autres plus ouvertes. Le contexte favorisant l'endogamie étant à la fois écologique et idéologique, le passage de l'endogamie à l'exogamie exigera donc un changement global peu probable.

Très souvent, la montée de l'exogamie est étroitement liée à un processus de minorisation de groupes qui ont connu des migrations qui les placent d'abord en situation de contacts, presque toujours dans des rapports majoritaires/minoritaires. $\mathrm{Si}$, au début, ces rapports sont plutôt tendus et conflictuels, ils passent assez rapidement à une phase d'acceptation et d'accommodation qui débouche sur la cohabitation. Après deux ou trois générations, la cohabitation provoque certaines formes d'acculturation qui conduiront éventuellement à l'assimilation à la majorité. Dans les modèles d'intégration des minorités, l'exogamie représente une des phases du processus d'assimilation. Ces phénomènes, imbriqués les uns dans les autres, se déroulent sur plusieurs générations. Les modèles explicatifs d'intégration des immigrants doivent faire ressortir la complexité et l'enchevêtrement des phénomènes sociodémographiques qui lient migration, exogamie et intégration. Dans notre analyse du passage de l'endogamie à l'exogamie à l'intérieur des communautés francophones minoritaires du Canada, nous tenterons de respecter cette exigence, soit celle de fournir une explication claire face à ce phénomène alambiqué. 
Pour ce faire, nous allons amorcer l'analyse par un survol historique rapide. Dans la très grande majorité des cas, le processus de minorisation des francophones canadiens se produit à la suite d'une émigration québécoise. En ce qui a trait aux Acadiens, la minorisation suit l'immigration anglophone, surtout en provenance des pays européens anglo-saxons. Toutefois dans les autres provinces, les francophones sont très souvent originaires d'un rang, d'un village ou d'une ville québécoise où les Canadiens-Français sont majoritaires. Par conséquent, l'émigration conduit dans bon nombre de cas ces derniers dans un rang, un village ou une ville où ils seront fort probablement encore majoritaires quant à l'unité locale (la communauté immédiate), mais ils seront minoritaires lorsque nous considérons l'environnement global de la région ou de la province. En guise d'exemple, les Canadiens-Français seront très majoritaires dans le village de Fauquier, dans le nord de l'Ontario, mais minoritaires dans la région de Cochrane et très minoritaires en Ontario.

Les mouvements migratoires des CanadiensFrançais du $\mathrm{XIX}^{\mathrm{e}}$ siècle et de la première moitié du $\mathrm{XX}^{\mathrm{e}}$ suivent habituellement le même scénario. Pourquoi ces derniers quittent-ils le Québec ? La réponse est fort complexe et certaines recherches se sont déjà penchées sur cette question ${ }^{20}$. De façon générale, les Canadiens-Français émigrent pour trouver un emploi stable, dans l'espoir d'une vie meilleure pour la famille. Pourtant, globalement ces derniers résistent habituellement à la migration interprovinciale. Ce-

\footnotetext{
${ }^{20}$ Roger Bernard, Le Travail et l'espoir. Migrations, développement économique et mobilité sociale. Québec/Ontario, 1900-1985, Hearst (Can.), Les éditions du Nordir, 1991, 396 p.; Rejean Lachapelle et Jacques Henripin, op. cit.
} 
pendant, s'ils doivent quitter leur milieu d'origine, ils préfèrent s'installer ailleurs au Québec et si possible dans le même canton. En dernière instance, s'ils doivent quitter le Québec, en autant que faire se peut, ils s'établiront près de la frontière québécoise.

Si l'incidence de l'exogamie des francophones minoritaires canadiens est liée aux mouvements migratoires interprovinciaux, l'explication touche à la fois l'économie, la démographie, la politique, le social, le culturel et le religieux en regard des sociétés d'origine et de destination, la migration étant elle aussi un phénomène démographique pluriel. Le but du présent article n'étant pas d'expliciter en profondeur les liens entre l'exogamie et la migration, la suite se propose de mettre en lumière d'autres facteurs ayant provoqué la montée de l'exogamie. Mais avant d'en arriver là, nous reviendrons en arrière, à une époque pas très lointaine, où l'endogamie était encore la norme sociale de l'organisation familiale des francophones minoritaires. En effet, même si en cette fin du $\mathrm{XX}^{\mathrm{e}}$ siècle, nous assistons à l'institutionnalisation de l'exogamie comme nouvelle forme d'organisation familiale, force est de reconnaître que les francophones ont résisté pendant plus d'un siècle, tel que le démontrent les premiers grands mouvements migratoires du XIX ${ }^{\mathrm{e}}$ siècle jusqu'au milieu du $\mathrm{XX}^{\mathrm{e}}$.

Ainsi, le premier grand facteur en regard de la force de l'endogamie, comme norme sociale à l'époque de la société rurale et traditionnelle, est essentiellement écologique, mais écologique au sens américain qui regroupe géographie, communauté et culture. Pour en saisir l'importance, nous devons reprendre un concept démographique crucial: les sentiers 
migratoires. Dans cette optique, il importe de mentionner que les Canadiens-Français qui quittent le Québec ont tendance à suivre des sentiers précis; les gens d'un village particulier à l'origine retrouvent souvent les leurs dans le village de destination. Il faut alors imaginer la migration comme une chaîne humaine entre l'origine et la destination; elle est rarement une marche vers l'inconnu: les sentiers sont habituellement bien battus. Dans les villages qu'ils habitent à l'arrivée et les paroisses qu'ils forment, ils réussissent assez souvent à demeurer majoritaires, à reproduire, à la destination, les conditions de vie qu'ils connaissaient à l'origine. Dans des villes plus populeuses, telles St-Catharines, Sudbury et Winnipeg, pour n'en nommer que quelques-unes, ils se regroupent autour d'une paroisse et, de ce fait, ils arrivent à former des quartiers francophones où ils seront plus ou moins minoritaires dans l'environnement immédiat ${ }^{21}$.

Dans ces nouvelles contrées, ils mettent sur pied les institutions nécessaires pour assurer la continuité et la survie: ils reproduisent en Ontario les structures qu'ils avaient connues au Québec. La famille est canadienne-française; elle regroupe des époux et des épouses francophones et catholiques; les enfants ont la langue maternelle et la religion des parents. L'école primaire de rang, de village ou de quartier, est elle aussi française et catholique, rassemblant une population écolière relativement homogène, composée essentiellement de jeunes Canadiens-Français catholiques. Les luttes pour obtenir ces écoles et les maintenir sont épiques et éloquentes; dans certaines régions, elles durent et

${ }^{21}$ Roger Bernard, Le Travail [...], op. cit. 
perdurent depuis plus d'un siècle. Ces institutions canadiennes-françaises favorisent sûrement l'endogamie. Toutefois, d'une région à l'autre et d'une province à l'autre, la complétude des services éducatifs en français varie grandement, de telle sorte que plusieurs jeunes francophones devront fréquenter l'école de langue anglaise. Cette dernière situation sera par conséquent plus favorable à l'exogamie qu'à l'endogamie.

En effet, au niveau secondaire, les choses se compliquent considérablement. Il faut d'abord retenir que durant la première moitié du $\mathrm{XX}^{\mathrm{e}}$ siècle, la fréquentation des écoles secondaires sera limitée et qu'une bonne proportion de ceux et de celles qui les fréquenteront se retrouveront dans les collèges ou les couvents privés, catholiques et français, les autres étant répartis dans les «high schools» publics et anglais. Il va sans dire que la complétude institutionnelle française favorise nettement l'endogamie, les francophones ne sentant pas le besoin de fréquenter des membres des autres groupes culturels pour former un foyer. Il en ira autrement pour ceux et celles qui seront obligés de fréquenter les écoles de langue anglaise puisqu'ils se retrouveront alors minoritaires à l'intérieur d'institutions de la majorité qui appelleront l'exogamie de manière implicite.

Mais pour bien comprendre la force de l'endogamie comme norme sociale, nous devons considérer plusieurs éléments de la culture canadienne-française, des éléments qui relèvent surtout de l'idéologie dominante de l'époque. Des premières migrations jusqu'au milieu du $\mathrm{XX}^{\mathrm{e}}$ siècle, la société canadiennefrançaise, incluant le Québec, est plutôt traditionnelle et catholique, caractérisée par son mode de vie 
rural, même si une bonne partie de la population habite la ville: ce sont des ruraux en ville; la mentalité rurale ne s'efface pas automatiquement avec le passage à la ville. Dans cette société, l'Église, l'école et la famille sont les principales institutions, alors que les valeurs sont intégralement religieuses. La religion catholique ultramontaine représente la valeur centrale de la culture canadienne-française, mais il nous faut reconnaître que la foi catholique et la langue française sont des éléments culturels étroitement liés l'un à l'autre, et qu'ils se trouvent dans des relations de complémentarité et de réciprocité, de telle sorte que les luttes pour la défense de la langue française deviennent facilement des combats de la fidélité à la religion catholique et inversement.

Dans ce contexte de culture religieuse traditionnelle, de société relativement fermée sur elle-même, dans une relative autarcie, l'exogamie est impensable et impraticable: choisir un conjoint de l'autre groupe, un Canadien-Anglais, c'est s'exposer au risque de perdre sa langue et de nier sa foi. Dans cet univers social et religieux, l'exogamie s'apparente à la transgression d'un tabou. Les représentations symboliques et les classifications identitaires sont explicites: le français est automatiquement associé au catholicisme et l'anglais au protestantisme. L'autre «étrange» sera identifié comme un protestant, même lorsqu'il s'agira d'un Irlandais catholique. Et si les obstacles démographiques, écologiques et culturels ne suffisent pas à modérer les élans amoureux illicites, les parents seront très réticents à accorder leur bénédiction à la future union qui ne trouvera pas grâce à leurs yeux, et l'Église catholique, dans le cas des conjoints non 
catholiques, introduira des embûches difficiles à contourner. Par ailleurs, si les futurs conjoints exogames procèdent en dépit des entraves, ils risquent la marginalisation et l'exclusion. Les barrières culturelles et religieuses se transforment en véritables empêchements qui limiteront très sérieusement le nombre de mariages mixtes à cette époque. L'endogamie est encore une norme sociale contraignante qui encadre le développement du sentiment amoureux.

En parallèle à ce frein à l'endoǵamie, nous devons considérer l'unilinguisme respectif des communautés anglophones et francophones au Canada. Il n'y a pas si longtemps, les contacts entre les deux grandes communautés linguistiques étaient limités parce que la grande majorité des Canadiens de langue anglaise ne connaissaient pas le français et, dans la même veine, une proportion importante de CanadiensFrançais ne connaissaient pas l'anglais. Les deux grandes "solitudes" étaient littéralement enfermées dans leur unilinguisme respectif et leur univers culturel singulier.

Cette période historique de repli sur soi sociétal va durer deux siècles: de la conquête à la Révolution tranquille. À partir de cette période de renouveau et d'ouverture, des bouleversements toucheront tous les aspects de la vie sociale et les ruptures identitaires marqueront l'évolution des communautés francophones et acadiennes. Cette conjoncture favorisera alors le passage de l'endogamie à l'exogamie.

\section{EXOGAMIE: UNE NOUVELLE STRUCTURE FAMILIALE}

Les changements culturels, démographiques, écologiques, sociolinguistiques et économiques que nous 
voyons poindre au début des années 1960 nous conduisent à une tout autre réalité. Le passage définitif d'une société traditionnelle, rurale et catholique, à une société moderne, urbaine et laïque, va modifier presque complètement le milieu de vie des communautés francophones minoritaires. Les communautés francophones majoritaires, surtout celles du Québec et du Nouveau-Brunswick, connaîtront également les grandes ruptures de la Révolution tranquille, mais les bouleversements du milieu de vie ne seront pas aussi dramatiques que dans les milieux minoritaires. De façon générale, plus les communautés francophones seront minoritaires, plus les changements démographiques et culturels seront profonds et déterminants; dans certains cas, ils seront irréversibles, rendant fragile la vitalité ethnolinguistique.

Trois types de migrations internes, en opposition aux migrations interprovinciales, vont ainsi marquer l'évolution des communautés francophones et acadiennes. La première migration interne a habituellement trait à la phase initiale de l'urbanisation, soit le passage du milieu rural à la ville, la plupart du temps dans la ville à proximité du milieu rural, alors que la deuxième phase d'urbanisation voit les francophones, souvent les enfants ou les petits-enfants des émigrants québécois, quitter les villages où ils sont majoritaires pour s'installer dans des villes plus grandes où ils seront vraiment minoritaires: c'est la phase avancée de l'urbanisation et de l'industrialisation. Le troisième mouvement est celui du déplacement vers la banlieue qui constitue la phase finale de la dislocation et de la minorisation.

Ces migrations provoquent alors des changements écologiques profonds et définitifs qui entraîneront 
des chambardements des milieux de vie et des réseaux de relations sociales. Ce nouveau contexte va favoriser l'exogamie au détriment de l'endogamie. Toutefois, si l'explication de la montée de l'exogamie est, en partie, écologique, il ne faut pas pour autant négliger les éléments idéologiques de l'explication.

À cet effet, pour une grande majorité des francophones, les processus de modernisation et de dislocation s'accompagnent presque toujours de processus de minorisation et d'anglicisation, et plus les processus de modernisation et de dislocation avancent, plus les processus de minorisation et d'anglicisation $s^{\prime}$ accentuent ${ }^{22}$. Les statistiques des recensements des trois dernières décennies montrent clairement la progression de la minorisation et de l'anglicisation. En quelques générations, la transition du monde rural et agricole, relativement isolé, à un monde urbain et industriel, va faire en sorte que bon nombre de Canadiens-Français se retrouvent désormais dans un environnement où le contact est maintenant continuel avec les anglophones et la langue anglaise qui domine le monde du travail, des affaires, des communications et des services. De la phase conflictuelle qui suit habituellement les premières migrations et la prise de possession du territoire, nous entrons maintenant dans une phase d'accommodement. Dans ce nouvel entourage culturel et social, la sphère réservée au français se limite souvent à la vie privée de la famille, alors que l'anglais s'impose comme langue

\footnotetext{
${ }^{22}$ Roger Bernard, Le Déclin d'une culture. Recherche, analyse et bibliographie. Francophonie hors Québec, 1980-1990, Ottawa, Fédération des jeunes Canadiens inc., 1990, 198 p.; id., Le Choc des nombres. Dossier statistique sur la francophonie canadienne, 1951-1986, Ottawa, Fédération des jeunes Canadiens inc., 311 p.; id., De Québécois d Ontarois, Ottawa, Les Éditions du Nordir, 1996 (1988), 179 p.
} 
publique de la vie quotidienne. C'est dans ce contexte que les francophones minoritaires doivent absolument être bilingues s'ils veulent se tailler une place, autant économique que sociale. Étant bilingues, ils ne cherchent pas le quartier francophone à tout prix, et il en est ainsi pour les écoles et les institutions qu'ils fréquentent et les services qu'ils reçoivent. La cohabitation conduit donc à un niveau d'accommodement linguistique et culturel élevé où l'anglais occupe la place dominante. Plusieurs parents francophones se verront alors contraints d'envoyer leurs enfants à l'école d'immersion en français (écoles conçues et organisées en fonction des besoins des jeunes anglophones) ou à l'école de langue anglaise si l'école de langue française n'est pas facilement accessible. Pour certains, cette solution de dernier recours sera catastrophique, mais pour d'autres, elle s'inscrira dans un processus normal de bilinguisation.

Comme suite à ces grands changements, nous pouvons sans conteste déduire que le nouvel environnement social et culturel va favoriser les fréquentations mixtes et, conséquemment, l'augmentation des taux d'exogamie. À cet égard, depuis quelques décennies les francophones sont de plus en plus minoritaires, de plus en plus dispersés, de plus en plus bilingues et, par conséquent, de plus en plus anglicisés; ces quatre conditions expliquent en partie, le passage de l'endogamie à l'exogamie.

Ce délestage du mode de pensée traditionnel, dans cette nouvelle conjoncture, fera en sorte que les dernières barrières culturelles et religieuses vont tomber en regard de l'exogamie. Outre ces changements, l'Église catholique, de par le souffle de tolérance et 
de renouveau de Vatican II, est plus ouverte aux rapprochements avec les autres Églises et, par le fait même, les mariages interreligieux, qui sont très souvent des mariages interculturels, deviennent moins problématiques puisque s'inscrivant dans l'esprit œcuménique d'ouverture à l'autre. De plus, la religion catholique, élément central de la culture canadiennefrançaise, est lentement dissociée des luttes pour la survie de la francophonie au Canada. En même temps qu'il y a laïcisation de l'ensemble de la société, il y a aussi laïcisation des combats pour la défense de la langue française. Les adages «la langue gardienne de la foi» et «la foi gardienne de la langue» perdent sens et pertinence. En l'espace de quelques décennies, la barrière religieuse disparaît replaçant la problématique de l'exogamie dans la sphère civile et la culture laïque.

La correspondance entre la communauté religieuse et la communauté culturelle n'est plus aussi étroite qu'auparavant. Alors que le Québec prend nettement et définitivement le virage de la francité comme projet sociétal, les communautés francophones et acadiennes, devant l'éclatement de la société canadiennefrançaise et la régionalisation des identités, prennent le virage d'une francité au sein de l'univers du bilinguisme pancanadien. Les combats pour assurer la vitalité de la langue et de la culture françaises passent par les politiques fédérales et provinciales du bilinguisme qui sont introduites à la suite de la publication du rapport de la Commission royale d'enquête sur le bilinguisme et le biculturalisme. Une nouvelle idéologie prend alors racine: le bilinguisme. Cependant l'idée du bilinguisme que nous voyons apparaître est différente de celle proposée par la Commission qui, à la limite, acceptait la notion d'un 
pays bilingue qui regroupe des communautés linguistiques et des individus qui ne sont pas nécessairement bilingues, mais qui ont à leur service des institutions qui peuvent répondre aux besoins et aux attentes des deux grandes communautés linguistiques. L'idée du bilinguisme qui prend forme propose, à la limite, que les individus soient, dans un premier temps, bilingues, c'est-à-dire, qu'ils maîtrisent si possible les deux langues et, dans un deuxième temps, biculturels, c'est-à-dire qu'ils appartiennent aux deux univers culturels, qu'ils vivent dans la pratique le biculturalisme, à la manière d'un Pierre ElliotTrudeau qui devient l'archétype du bilinguisme et du biculturalisme. Dans ce nouveau contexte, il faut réaliser que la francité est rattachée au bilinguisme (certains diraient: à la remorque du bilinguisme), dans une relation de dépendance et de réciprocité, comme elle était autrefois rattachée à la religion catholique dans le même type de relation de dépendance et de sujétion. Pour un grand nombre de francophones minoritaires, c'est le bilinguisme qui devient effectivement l'élément central de la culture collective et de l'identité individuelle. La francité est valorisée parce qu'elle est greffée au bilinguisme et c'est par l'entremise du bilinguisme qu'elle devient une valeur culturelle; elle n'est pas en elle-même une valeur centrale de la culture francophone minoritaire.

Dans ce contexte social et idéologique particulier qui se développe à la fin des années 1960, l'exogamie n'est plus un tabou. Au contraire, le mariage mixte permet de réaliser un rêve et d'atteindre un idéal: former un foyer bilingue, dans un pays bilingue, et avoir des enfants qui seront bilingues et biculturels, dans l'espoir qu'ils maîtriseront l'anglais, qu'ils pour- 
TRANSFERTS LINGUISTIQUES ET ANGLICISATION

ront l'écrire et le parler comme les anglophones, sans succomber au piège du transfert linguistique et de l'anglicisation.

Ainsi, si le contexte sociétal conduit naturellement à l'assimilation, les parents exogames sont nombreux à croire qu'ils sauront résister aux forces de l'acculturation. De plus, ils croient souvent, à tort, que le maintien du français dans des conditions adverses est une question de volonté du couple et de convictions personnelles, qu'ils réussiront où les autres ont échoué.

Tous les éléments sont désormais en place pour le passage de l'endogamie à l'exogamie. Les barrières culturelles, religieuses et linguistiques sont tombées; les facteurs démographiques, écologiques et sociaux favorisent une cohabitation plus poussée; les nouvelles valeurs d'ouverture à l'autre, de métissage identitaire et de mondialisation des perspectives propres à la postmodernité encouragent la suppression des frontières ethniques et le partage des institutions sociales. Le pluralisme et le multiculturalisme sont à l'honneur. L'exogamie n'est plus sacrilège; au contraire, elle permet de vivre une situation de bilinguisme intégral et de biculturalisme plus engagé, le grand rêve de nombreux francophones qui aspirent à se définir par une appartenance à deux cultures.

Le passage de la prédominance de l'endogamie, comme forme d'organisation familiale à l'intérieur des communautés francophones et acadiennes, à l'institutionnalisation de l'exogamie est fort probablement irréversible. En quelques décennies, ces communautés sont passées d'une culture religieuse très contraignante, hermétique et refermée sur elle-même, 
à une culture médiatique éclatée et ouverte. Les tendances qui sont décrites sont appelées à s'accentuer durant les prochaines années; elles vont sûrement créer un contexte encore plus favorable à une augmentation des taux d'exogamie.

En analysant les principales données démographiques des communautés francophones et acadiennes, nous pourrions, pour les prochaines années, brosser le tableau suivant: la minorisation et la dispersion des francophones risquent de s'accentuer dans toutes les provinces canadiennes à l'exception du Québec; les transferts linguistiques du français vers l'anglais vont continuer d'augmenter; les pyramides des âges de la population francophone s'inversent; le nombre de jeunes francophones diminue d'une génération à l'autre; les indices de fécondité demeureront faibles et inférieurs à la moyenne canadienne ${ }^{23}$. Conséquemment, il en résulte «une tendance extrêmement forte de la reproduction linguistique vers la baisse ${ }^{24}$ ". En considérant que plus les francophones sont minoritaires, plus ces tendances sont prononcées, il appert que nous assisterons à une montée de l'exogamie, et il est tout à fait inattendu que, dans un avenir rapproché, nous puissions observer une régression de ce phénomène.

L'exogamie représente donc aujourd'hui une réalité sociale avec laquelle les communautés francophones et acadiennes doivent composer. Et si elle est là pour rester, il faut poser la question épineuse suivante, à savoir: est-ce que l'exogamie est un phéno-

\footnotetext{
${ }^{23}$ Roger Bernard, Le Travail [...], op. cit.

${ }^{24}$ Charles Castonguay, «Évolution récente de l'assimilation linguistique au Canada», in Langue, espace, société. Les Variétés du français en Amérique du Nord, Québec, Les Presses de l'Université Laval, 1994, pp. 277-311.
} 
mène inquiétant en regard de la vitalité linguistique et culturelle des communautés francophones et acadiennes? En d'autres mots, quels sont les liens entre l'exogamie et l'anglicisation?

\section{III - EXogamie et anglicisation}

Les liens entre l'exogamie et l'anglicisation sont complexes, les relations de causalité pouvant s'inverser d'une situation à l'autre. De plus, en reprenant les distinctions de Castonguay, il faut prendre en considération qu'il y a trois grandes formes d'assimilation, soit l'assimilation individuelle, l'assimilation intergénérationnelle et l'assimilation collective qui peuvent être associées à l'exogamie de diverses façons ${ }^{25}$.

À cet égard, Castonguay ${ }^{26}$ distingue deux situations selon des taux différents d'exogamie qui sont associés à des sociétés tantôt majoritaires et tantôt minoritaires. Il affirme que dans certains cas l'exogamie conduit inévitablement à l'assimilation individuelle des membres francophones, et cela même si le taux d'exogamie est faible à l'intérieur de la communauté concernée, tel qu'on le retrouve au Québec et dans certaines régions du NouveauBrunswick. Dans cette perspective, il se pourrait très bien que l'effet de l'exogamie en regard de l'assimilation collective soit déterminant. Donc, en situation de majorité et de faible exogamie, les taux d'anglicisation seront normalement faibles, mais ils seront, en grande partie, expliqués par l'exogamie. Par ailleurs, dans d'autres situations où les taux

${ }^{25}$ Charles Castonguay, «Mesure de l'assimilation linguistique au moyen des recensements», Recherches sociographiques, vol. XXXIV, n ${ }^{\circ} 1$, p. 47. ${ }^{26} I d$., "Évolution récente [...]", op. cit. 
d'exogamie sont très élevés, donc dans les milieux très minoritaires, nous sommes portés à croire que l'exogamie est vraiment l'élément essentiel qui explique l'assimilation. Pourtant, dans ce cas précis, il ne serait pas surprenant de réaliser que l'anglicisation a précédé le mariage mixte, de telle sorte que nous pourrions probablement considérer qu'il ne s'agit pas d'un mariage mixte, même si un des partenaires est d'origine française. Les deux phénomènes sont étroitement liés l'un à l'autre, dans une covariation qui peut être linéaire, mais le mariage mixte n'est pas l'élément qui explique l'étendue de l'assimilation collective. Nonobstant cette particularité, nous retrouvons d'autres situations où l'exogamie précède l'assimilation et où elle explique en grande partie les taux élevés d'anglicisation.

À la lumière des conclusions de cet auteur, nous sommes en mesure de réaliser que les liens entre l'exogamie et l'anglicisation sont fort complexes et nous tenterons de les expliciter dans la dernière partie de ce travail. Toutefois, avant de nous pencher sur cette problématique, nous allons analyser les données relatives aux taux d'anglicisation des partenaires francophones des mariages mixtes par le biais de la langue d'usage au foyer et les taux d'anglicisation des enfants issus de ces mariages de même que par l'identification de la langue maternelle transmise par les parents à l'intérieur des foyers endogames et exogames.

\section{ANGLICISATION DES PARTENAIRES FRANCOPHONES}

Les données du sixième tableau sont particulièrement révélatrices. La première grande constatation, lorsque nous considérons l'ensemble des commu- 
nautés francophones hors Québec, est à l'effet que 9 époux sur 10 de langue maternelle française utilisent habituellement l'anglais à la maison en situation d'exogamie, alors que cette proportion est de 8 sur 10 pour les épouses. Dans les deux cas, les taux d'anglicisation sont très élevés, mais les époux sont plus anglicisés que les épouses. Même dans les provinces où les taux d'exogamie sont plus faibles que la moyenne, la langue d'usage des partenaires francophones est habituellement l'anglais. Il n'y a qu'au Québec que les taux d'anglicisation sont relativement faibles: $15 \%$ pour les épouses francophones et $30 \%$ pour les époux. Au Nouveau-Brunswick, où les taux d'exogamie sont inférieurs à $15 \%$, nous retrouvons tout de même plus de $60 \%$ des épouses francophones qui ont l'anglais comme langue d'usage au foyer, comparativement à plus de $70 \%$ des époux. En ce qui a trait à l'Ontario, les taux d'exogamie oscillent autour de $40 \%$, mais $80 \%$ des épouses francophones et $90 \%$ des époux ont l'anglais comme langue d'usage. Une autre constatation concerne les taux. d'anglicisation qui sont relativement stables d'un recensement à l'autre lorsque nous comparons 1986 par rapport à 1971. Dans certains cas, le taux d'anglicisation est plus élevé en 1986 qu'en 1971; dans d'autres cas, c'est le contraire. Il est à noter toutefois que les diminutions sont plus importantes et plus fréquentes en 1986 par rapport à 1971, lorsque les francophones sont moins minoritaires et que les taux d'exogamie sont plus bas. 
TABLEAU 6

Taux d'anglicisation des partenaires de langue maternelle française dans les mariages exogames, provinces et Canada, 1971 et 1986.

\begin{tabular}{lrrrr}
\hline & \multicolumn{2}{c}{ Époux de langue } & \multicolumn{2}{c}{ Épouse de langue } \\
maternelle française & \multicolumn{2}{c}{ maternelle française } \\
Provinces & 1971 & 1986 & 1971 & 1986 \\
Colombie-Britannique & 97,9 & 97,4 & 94,6 & 92,9 \\
Alberta & 96,8 & 97,0 & 95,7 & 93,4 \\
Saskatchewan & 97,3 & 96,9 & 95,5 & 96,6 \\
Manitoba & 95,1 & 94,1 & 91,6 & 92,5 \\
Ontario & 92,8 & 90,0 & 87,7 & 80,1 \\
Québec & - & 29,8 & - & 14,5 \\
Nouveau-Brunswick & 82,6 & 70,8 & 77,5 & 62,0 \\
Île-du-Prince-Édouard & 95,8 & 100,0 & 91,3 & 91,3 \\
Nouvelle-Écosse & 93,6 & 92,3 & 90,2 & 86,3 \\
Terre-Neuve & 91,4 & 79,6 & 86,7 & 86,5 \\
Canada (sans le Québec) & - & 88,4 & & 82,8 \\
Canada & - & 70,3 & & 54,2 \\
\hline
\end{tabular}

SOURCE $^{27}$

Cependant, pour les observateurs avertis, l'étendue de l'anglicisation n'a rien de très surprenant. Dans la très grande majorité des cas, le partenaire francophone est bilingue, alors que le partenaire anglophone est habituellement unilingue. Lorsqu'une

${ }^{27}$ Recensement du Canada de 1991. 
personne unilingue se trouve en situation d'échange avec une personne bilingue, comme c'est très souvent le cas dans les familles mixtes, la personne unilingue va imposer la langue d'usage, parce que la personne bilingue peut facilement, et souvent indifféremment, utiliser l'une ou l'autre langue. Or, si la compétence linguistique respective des conjoints influence le choix de la langue d'usage, il ne faut pas perdre de vue que le contexte global de la famille exogame, dans un milieu anglo-dominant la plupart du temps, va exercer des pressions en faveur de l'utilisation de l'anglais.

\section{ANGLICISATION DES ENFANTS}

Les données du septième et du huitième tableau sont également éloquentes: la majorité des enfants issus des mariages mixtes s'anglicisent. En effet, lorsque nous considérons la langue maternelle de ces derniers, force est de constater que c'est surtout la langue anglaise qui est transmise dans les foyers mixtes, ce qui témoigne d'un cas très clair d'assimilation intergénérationnelle. Et ce qui frappe en premier lieu, c'est l'ampleur de cette assimilation.

À l'exception du Québec, la très grande majorité des enfants vivant dans une famille mixte ont l'anglais comme langue maternelle unique: dans les familles où le père est francophone et la mère anglophone, approximativement 9 enfants sur 10 de moins de 18 ans ont l'anglais comme langue maternelle unique tandis qu'au Nouveau-Brunswick cette proportion baisse à 7 sur 10. Au Québec, la proportion est inférieure à 5 sur 10 , mais toutefois le pourcentage de ceux qui ont l'anglais comme langue maternelle est plus élevé que ceux qui ont le français 
comme langue maternelle, soit $43,9 \%$ comparativement à $40 \%$, presque tous les autres cas $(16 \%)^{28}$ ayant à la fois l'anglais et le français comme langues maternelles.

\section{TABLEAU 7}

Enfants de moins de 18 ans de langue maternelle françalse vivant dans une famille époux-épouse selon la langue maternelle de l'époux et la langue maternelle de l'épouse (endogamie et exogamie), provinces et Canada, 1991 (pourcentages).

\begin{tabular}{lcccc}
\hline & \multicolumn{2}{c}{ Endogamie } & \multicolumn{2}{c}{ Exogamie } \\
$\begin{array}{l}\text { Provinces } \\
\text { et Canada }\end{array}$ & $\begin{array}{c}\text { Mère an. } \\
\text { Père an. }\end{array}$ & $\begin{array}{c}\text { Père fr. } \mathrm{fr} . \\
\text { Colombie-Britannique }\end{array}$ & $\begin{array}{c}\text { Mère an. } \\
\text { Père fr. }\end{array}$ & $\begin{array}{c}\text { Mère fr. } \\
\text { Pèr. }\end{array}$ \\
Alberta & 0,03 & 67,4 & 2,5 & 8,5 \\
Saskatchewan & 0,03 & 75,0 & 3,0 & 9,5 \\
Manitoba & 0,02 & 64,6 & 3,6 & 6,1 \\
Ontario & 0,04 & 85,8 & 6,3 & 14,6 \\
Québec & 0,06 & 93,3 & 8,8 & 22,5 \\
Nouveau-Brunswick & 1,17 & 99,7 & 40,0 & 57,9 \\
Île-du-Prince-Édouard & 0,1 & 98,3 & 21,0 & 31,5 \\
Nouvelle-Écosse & 0,04 & 85,8 & 7,1 & 11,2 \\
Terre-Neuve & 0,04 & 84,4 & 3,9 & 11,9 \\
Yukon et T.N.-O. & 0,09 & 82,5 & 7,3 & 11,7 \\
Canada & 0,07 & 98,9 & 16,6 & 29,0 \\
\hline
\end{tabular}

SOURCE $^{29}$

${ }^{28}$ Ce pourcentage est tiré des données du recensement du Canada de 1991. ${ }^{29}$ Recensement du Canada de 1991. 
TABLEAU 8

Enfants de moins de 18 ans de langue matemelle anglaise unique vivant dans une famille époux-épouse selon la langue maternelle de l'époux et la langue maternelle de l'épouse, provinces et Canada, 1991 (pourcentages).

\begin{tabular}{|c|c|c|c|c|}
\hline \multirow[b]{2}{*}{$\begin{array}{l}\text { Provinces et } \\
\text { Canada }\end{array}$} & \multicolumn{2}{|c|}{ Endogamie } & \multicolumn{2}{|c|}{ Exogamie } \\
\hline & $\begin{array}{l}\text { Mère an. } \\
\text { Père an. }\end{array}$ & $\begin{array}{l}\text { Mère fr. } \\
\text { Père fr. }\end{array}$ & $\begin{array}{l}\text { Mère an. } \\
\text { Père fr. }\end{array}$ & $\begin{array}{l}\text { Mère fr. } \\
\text { Père an. }\end{array}$ \\
\hline Colombie-Britannique & 99,8 & 27,3 & 92,5 & 82,4 \\
\hline Alberta & 99,8 & 21,1 & 93,2 & 83,7 \\
\hline Saskatchewan & 99,9 & 31,4 & 93,6 & 89,2 \\
\hline Manitoba & 99,7 & 12,4 & 89,5 & 78,9 \\
\hline Ontario & 99,7 & 5,3 & 84,3 & 66,5 \\
\hline Québec & 97,5 & 0,2 & 43,9 & 24,3 \\
\hline Nouveau-Brunswick & 99,7 & 1,5 & 71,8 & 57,7 \\
\hline Île-du-Prince-Édouard & 99,8 & 15,0 & 88,6 & 82,9 \\
\hline Nouvelle-Écosse & 99,9 & 12,3 & 89,0 & 79,7 \\
\hline Terre-Neuve & 99,9 & 11,1 & 90,4 & 75,0 \\
\hline Yukon et T.N.-O. & 99,7 & 17,5 & 88,4 & 80,0 \\
\hline Canada & 99,7 & 0,8 & 74,8 & 59,2 \\
\hline
\end{tabular}

Le transfert linguistique du français à l'anglais n'est pas l'apanage des seuls couples exogames. En consultant le tableau 8 , nous remarquons qu'en situation très minoritaire, les familles endogames françaises

${ }^{30}$ Recensement du Canada de 1991. 
éprouvent certaines difficultés à transmettre le français comme langue maternelle d'une génération à l'autre. En guise d'exemple, $21 \%$ des enfants des familles endogames françaises de l'Alberta ont l'anglais comme langue maternelle et, en Saskatchewan, la proportion monte à $31 \%$. Il va donc de soi que, plus les francophones sont majoritaires, plus les couples endogames francophones résistent au transfert linguistique. Pour illustrer ce phénomène, notons qu'en Ontario seulement $5 \%$ des enfants des couples endogames francophones, ont l'anglais comme langue maternelle; au Nouveau-Brunswick, cette proportion baisse à $1,5 \%$ alors qu'au Québec elle est infime, soit $0,2 \%$. Il en est autrement pour les familles endogames anglophones. En effet, près de $100 \%$ des enfants de ces familles et ce, dans toutes les provinces canadiennes exception faite du Québec, ont l'anglais comme langue maternelle unique (les pourcentages varient effectivement de 99,7 à $99,9 \%$ ). Pour le Québec, cette proportion se situe à $97,5 \%$ en considération de $1 \%$ des enfants qui ont le français comme langue maternelle.

La deuxième grande constatation est à l'effet que les mères francophones réussissent mieux que les pères francophones à transmettre le français comme langue maternelle unique aux enfants, la différence étant approximativement de $15 \%$ pour l'ensemble du Canada. Le pouvoir de rétention de la langue maternelle des femmes est plus marqué dans les milieux moins minoritaires, cette différence étant plus grande au Québec que dans les autres provinces et ainsi de suite. Dans la plupart des provinces canadiennes, dans les foyers où la mère est francophone et le père anglophone, approximativement 8 enfants 
sur 10 de moins de 18 ans ont l'anglais comme langue maternelle unique.

Dans toutes les situations d'exogamie réunissant un parent francophone et un parent anglophone que nous avons étudiées, il y a seulement une conjoncture dans une province où le français s'impose véritablement aux enfants comme langue maternelle. Lorsque le mariage mixte réunit une mère francophone et un père anglophone et que cette famille exogame vit au Québec, 57,9\% des enfants ont le français comme langue maternelle unique. Dans toutes les autres situations, dans toutes les autres provinces, c'est l'anglais qui prédomine nettement comme langue maternelle des enfants.

En consultant les données du tableau 9 qui présente les différentes langues maternelles des enfants de moins de 18 ans vivant soit dans des familles endogames ou exogames, nous pouvons observer un phénomène intéressant lié au bilinguisme: la très grande majorité des enfants ont soit l'anglais ou le français comme langue maternelle et très peu ont à la fois les deux langues comme langues maternelles. En effet, seulement $11 \%$ des enfants d'une mère française et d'un père anglais possèdent simultanément le français et l'anglais comme langue maternelle. Dans tous les autres cas, cette proportion est inférieure à $7 \%$. 


\section{TABLEAU 9}

Langue maternelle des enfants de moins de 18 ans vivant dans une famille époux-épouse selon la langue maternelle de l'épouse et la langue maternelle de l'époux (endogamie et exogamie), Ontario, 1991 (pourcentages).

\begin{tabular}{lrrrrr}
\hline $\begin{array}{l}\text { Langue } \\
\text { maternelle } \\
\text { de l'enfant }\end{array}$ & \multicolumn{2}{c}{ Endogamie } & \multicolumn{2}{c}{ Exogamie an. } \\
Père an. & $\begin{array}{r}\text { Mère fr. } \\
\text { Père fr. }\end{array}$ & $\begin{array}{r}\text { Mère an. } \\
\text { Père fr. }\end{array}$ & $\begin{array}{r}\text { Mère fr. } \\
\text { Père an. }\end{array}$ \\
Anglais & 99,72 & 5,32 & 84,32 & 66,46 \\
Français & 0,06 & 93,31 & 8,84 & 22,48 \\
Français et anglais & 0,15 & 1,29 & 6,78 & 10,99 \\
Français et autres & 0,00 & 0,00 & & 0,03 \\
Autres & 0,06 & 0,07 & 0,05 & 0,07 \\
Total & $100 \%$ & $100 \%$ & $100 \%$ & $100 \%$ \\
N & $(1301405)$ & $(58695)$ & $(36935)$ & $(37905)$ \\
\hline
\end{tabular}

SOURCE $^{31}$

À la lumière de l'ensemble des données qui précèdent, il est évident que plus le milieu est minoritaire, plus les taux d'exogamie sont élevés, et plus les taux d'anglicisation des parents et des enfants sont élevés. Il faut toutefois rappeler qu'il s'agit seulement d'une covariation puisque ces données descriptives (groupées) ne permettent pas d'établir des liens de causalité, même s'il est fort probable que ces derniers existent. Cette prochaine partie, dans l'optique de ce qui a été exploré précédemment, se propose d'expliciter davantage les liens entre l'exogamie et l'anglicisation.

${ }^{31}$ Recensement du Canada de 1991. 


\section{UNE RELATION EXPLICATIVE COMPLEXE}

D'après les données des recensements du Canada que nous avons présentées et analysées, il apparaît clairement que certaines formes d'anglicisation, individuelle et collective, sont étroitement liées à l'exogamie entre francophones et anglophones. Néanmoins, affirmer et démontrer que les liens sont étroits ne veut pas dire que nous établissons des liens de causalité entre ces deux phénomènes. Pour expliciter ces liens, nous tenterons de répondre à la question suivante: est-ce que l'exogamie est une cause importante de l'assimilation linguistique des communautés francophones minoritaires du Canada? Pour ce faire, nous reprendrons les principales thèses de deux chercheurs chevronnés: Réjean Lachapelle et Charles Castonguay.

Dans son analyse de l'exogamie dans les populations francophones en situation minoritaire au Canada, Lachapelle ${ }^{32}$ résume de la façon suivante les données de 1991:

- «La fréquence de l'exogamie chez les francophones est inversement liée à la proportion qu'ils représentent dans le milieu.

- Le taux d'anglicisation est fonction de la fréquence de l'exogamie dans le milieu.

- L'exogamie est en augmentation chez les jeunes francophones, mais l'anglicisation a plutôt tendance à diminuer.

- La transmission de la mère aux enfants du français langue maternelle est fonction des attributs linguistiques du père.»

Dans un premier temps, Lachapelle démontre clairement que le taux d'exogamie des francophones est

${ }^{32}$ Réjean Lachapelle, L'Exogamie [...], op. cit. 
inversement proportionnel à leur poids démographique et que cette relation devient exponentielle lorsque la proportion de francophones dans les divisions de recensement est inférieure à $10 \%$. En considérant que nous assistons à un processus de plus en plus prononcé de minorisation et de dispersion des francophones, il est clair que ces tendances démographiques, associées aux autres facteurs qui favorisent une cohabitation linguistique et culturelle plus poussée, vont contribuer à une augmentation continue des taux d'exogamie chez les francophones durant les prochaines décennies. Outre cela, Lachapelle soutient, données à l'appui, que le taux d'anglicisation des francophones est fonction de la fréquence de l'exogamie dans le milieu de vie; cette relation est presque linéaire, et Lachapelle affirme que chaque unité d'augmentation du taux d'exogamie au Canada sans le Québec est accompagnée d'une augmentation proportionnelle du taux net de transfert linguistique. Deux grandes difficultés guettent les analystes qui tentent d'établir des liens de causalité entre l'exogamie et l'anglicisation: d'abord, ces deux phénomènes sont fortement corrélés; ensuite, les facteurs explicatifs de l'exogamie sont en grande partie les facteurs explicatifs de l'anglicisation. Dans un modèle de causalité, il faudrait considérer l'anglicisation comme la variable dépendante, l'exogamie comme une variable intermédiaire et les autres facteurs explicatifs comme des variables indépendantes et intermédiaires. C'est probablement en raison de ces difficultés méthodologiques et statistiques que les analystes n'ont pas encore construit de diagrammes de causalité pour établir la pondération de ces phénomènes en regard de l'explication des taux d'assimilation. 
Nonobstant ces difficultés méthodologiques et statistiques, nous pouvons trouver chez Lachapelle et Castonguay des éléments de réponse. En effet, l'analyse de Lachapelle ${ }^{33}$ établit que la transmission du français langue maternelle varie sensiblement d'une situation d'endogamie à une situation d'exogamie. La différence est remarquable. En effet, lorsque les deux parents sont francophones, la transmission du français langue maternelle aux enfants se fait dans $90 \%$ des cas, alors que cette proportion baisse à $20 \%$ lorsque la mère est francophone et que le père est anglophone. Cette proportion diminue si le père est francophone et la mère anglophone; toutefois, dans le cas précédent, si l'époux anglophone peut parler le français, la proportion augmente considérablement: la transmission du français langue maternelle de l'épouse aux enfants se produit dans la moitié des familles mixtes.

Pour tenter d'aller plus en profondeur dans les relations causales qui relient l'exogamie et l'assimilation linguistique chez les minorités canadiennes-françaises, nous devons revoir les nombreuses et excellentes études statistiques de Charles Castonguay qui reprennent les données des recensements de 1971,1981 et 1986 . Les résultats des recherches de Castonguay corroborent ceux de Lachapelle: les taux d'exogamie et de transferts linguistiques sont fortement corrélés, ils sont inversement proportionnels au poids démographique des différentes minorités provinciales canadiennesfrançaises, et ils augmentent à travers le temps en s'amplifiant d'une génération à l'autre, compromettant ainsi les capacités de reproduction linguistique des communautés francophones minoritaires.

${ }^{33}$ Rejean Lachapelle, ibid. 
Par ailleurs, si Castonguay reconnaît et identifie une forte relation entre l'exogamie et l'anglicisation, il invite néanmoins les analystes à la prudence en ce qui a trait aux relations de causalité. Au Québec, si moins de la moitié des transferts linguistiques s'explique par l'exogamie, il faut tout de même accepter l'idée qu'une grande partie de ces transferts s'explique par les mariages mixtes, même si les taux d'exogamie et de transferts sont plutôt faibles. Dans cette perspective, Castonguay identifie le mariage mixte comme un initiateur "primordial du transfert à l'anglais comme langue au foyer ${ }^{34}{ }^{\text {}}$ et, fait surprenant, plus les minorités sont résistantes à l'anglicisation, plus le mariage mixte est un élément déclencheur de l'anglicisation et plus la valeur explicative de l'exogamie en regard de l'assimilation est élevée. Cette valeur explicative est plus élevée au Québec qu'au Nouveau-Brunswick et plus élevée au NouveauBrunswick qu'en Ontario et ainsi de suite. Les autres facteurs explicatifs de l'anglicisation, notamment la minorisation, la dispersion et les migrations sont absents, de telle sorte que, même si les taux d'anglicisation sont faibles, l'exogamie est une variable explicative très importante. Chez les minorités francophones à l'extérieur du Québec, les taux d'exogamie et d'anglicisation sont très élevés et fortement corrélés, mais les personnes exogames vivant dans des familles exogames comptent pour moins de la moitié des transferts linguistiques. Plus les francophones sont minoritaires, plus l'exogamie prend une "valeur explicative dégressive" en regard de l'assimilation. En effet, dans cette situation, la minorisation, la dispersion, la faible fécondité et les

\footnotetext{
${ }^{34}$ Charles Castonguay, Exogamie et anglicisation [...], op. cit., p. 21
} 
migrations internes et internationales sont des facteurs importants dans l'explication des transferts linguistiques. Souvent, le transfert linguistique précède le mariage mixte et, alors, la relation de causalité s'inverse.

En dépit des remarques et des nuances qui précèdent en ce qui a trait à la relation de causalité entre l'exogamie et l'anglicisation, la réponse à la question: est-ce que l'exogamie est une cause de l'anglicisation? est affirmative. Ceci parce que plus les francophones sont minoritaires, plus les taux d'exogamie sont élevés et plus l'anglais s'impose comme langue d'usage dans les foyers mixtes. Cependant, d'autres facteurs expliquent en même temps les taux d'anglicisation élevés. Même dans les situations où les francophones sont majoritaires et résistent à l'assimilation, l'exogamie demeure un facteur qui déclenche et explique, en grande partie, les transferts linguistiques. Ainsi, dans les foyers mixtes, il appert que l'anglais domine dans l'aire linguistique familiale et, naturellement, les enfants adoptent, comme langue maternelle et langue d'usage, la langue d'usage des parents qui s'avère être, dans la très grande majorité des cas, l'anglais. À la lecture des données statistiques que nous avons analysées et en acceptant l'analyse des spécialistes présentée, nous sommes contraints de reconnaître que l'exogamie est une des causes de l'assimilation des minorités francophones du Canada.

\section{Conceusion}

Au Canada, comme le démontrent assez clairement les données analysées et les études réalisées au cours des dernières années, les transferts linguistiques 
et l'anglicisation comptent parmi les enjeux de l'exogamie. De façon générale, plus la vitalité ethnoculturelle des minorités canadiennes-françaises et acadiennes est faible, plus les enjeux de l'exogamie sont grands.

Dans le contexte de la société canadiennefrançaise traditionnelle, l'exogamie est impensable et impraticable: choisir un conjoint anglophone, c'est s'exposer au risque de perdre sa langue et d'en arriver à devoir nier sa foi catholique. Avec la Révolution tranquille, la laïcisation de la société et les autres changements liés aux processus de modernisation (qui conduisent à diverses formes de dispersion et de minorisation tout en favorisant la cohabitation institutionnelle et l'accommodement culturel), l'endogamie ne sera plus une norme sociale contraignante. Ces bouleversements et ces ruptures favoriseront la montée de l'exogamie. Toutefois, l'ampleur de l'exogamie varie en fonction des conditions démographiques: en situation très minoritaire, le taux d'exogamie est supérieur au taux d'endogamie; en situation majoritaire, l'exogamie est un phénomène marginal.

Pour répondre à la question visant à savoir si l'exogamie est une cause de l'anglicisation, il faut d'abord reconnaître qu'il ne s'agit pas du seul facteur explicatif. Par contre, à la lumière de l'analyse des données statistiques, nous devons reconnaître que l'exogamie est une des causes de l'assimilation des minorités francophones du Canada incluant le fait que, même au Québec, en situation majoritaire, l'exogamie explique, en partie les transferts linguistiques vers l'anglais. 